\title{
Sentinel node biopsy in breast cancer patients treated with neoadjuvant chemotherapy
}

\author{
YOSUKE TANAKA ${ }^{1}$, HIRONORI MAEDA $^{2}$, YASUHIRO OGAWA $^{3}$, AKIHITO NISHIOKA $^{3}$, \\ SATOSHI ITOH ${ }^{3}$, KEI KUBOTA ${ }^{3}$, HIRONOBU UE ${ }^{3}$, KIMIKO NAKATANI $^{3}$ and SHIRO SASAGURI ${ }^{2}$ \\ ${ }^{1}$ Operation Center, and Departments of ${ }^{2}$ Surgery II and ${ }^{3}$ Radiology, \\ Kochi University Hospital, Kochi Medical School, Kochi University, Japan
}

Received October 18, 2005; Accepted December 22, 2005

\begin{abstract}
A sentinel node biopsy (SNB) has been proved to be an accurate method to estimate the axillary lymph node status as a replacement for axillary lymph node dissection (AxLND) in patients with early breast cancer who have not been treated with neoadjuvant chemotherapy (NAC). We examined the feasibility and accuracy of performing SNB after NAC. Seventy breast cancer patients treated with NAC were enrolled in the current study during the period between March 2001 and June 2005. NAC performed preoperatively consisted of three to four times of CAF chemotherapy. Moreover, intra-arterial (subclavian artery and internal mammary artery) infusion of epirubicin and 5-fluorouracil was performed in addition to systemic CAF chemotherapy once to three times in patients with large breast tumors or bulky axillary lymph node metastases. The sentinel nodes were successfully identified in 63 out of 70 patients (identification rate: $90 \%$ ). The mean number of sentinel nodes removed per patient was 1.5 (range 1-6). Of the 43 patients in whom AxLND was performed after the sentinel nodes were identified, $19(44.2 \%)$ had positive sentinel nodes. In 8 of those 19 patients, the sentinel node was the only cancer positive lymph node. Among the 24 patients who had negative sentinel nodes it was found that one patient had a confirmed false negative result, thus yielding a false negative rate of $5 \%$, and a sensitivity of $95 \%$. There was no false negative patient who had a clinically negative lymph node status (N0) before NAC (17 patients), whereas the false negative rate was $6.3 \%$ in the subgroup of patients with a clinically positive lymph node status (N1, N2) before NAC (26 patients). As a result, SNB after NAC is thus considered to be able to effectively predict the axillary lymph node status in patients with a clinically negative lymph node status before NAC.
\end{abstract}

Correspondence to: Dr Yosuke Tanaka, Operation Center, Kochi University Hospital, Kochi Medical School, Kochi University, Oko-cho, Nankoku-shi, Kochi-Prefecture 783-8505, Japan

E-mail: tanakayo@med.kochi-u.ac.jp

Key words: sentinel node biopsy, neoadjuvant chemotherapy, breast cancer

\section{Introduction}

Axillary lymph node dissection (AxLND) has been routinely performed in breast cancer operations, because the regional lymph node status is known to be the most important prognostic factor in breast cancer, as well as being an important decision factor in selecting the optimal postoperative adjuvant therapy. However, there is no evidence that AxLND improves the prognosis of breast cancer patients, and the main therapeutic role of AxLND is thus considered to be to achieve axillary local control. A sentinel node biopsy (SNB) has been rapidly introduced in many institutes to determine the staging for the axilla of breast cancer patients who were supposed to have no axillary lymph node metastasis as a replacement for AxLND which is associated with a higher morbidity than SNB.

Patients who showed a complete pathological response (pCR) after neoadjuvant chemotherapy (NAC) proved to have an excellent prognosis (1). As a result the number of institutes that use NAC is increasing. The biggest advantage of NAC is to clarify the cancer characteristics of sensitivity to anti-cancer agents in vivo, which is valuable information for selecting the optimal anti-cancer agents for each breast cancer patient. In this context, SNB after NAC may thus enable us to avoid AxLND in cases that positively respond to NAC. However, in patients treated with NAC, SNB has not been recognized as a replacement for AxLND, because NAC may affect the lymphatic drainage and therefore hamper sentinel node detection. There is still limited information on the feasibility and accuracy of SNB after NAC.

The objective of the current study was to investigate the feasibility and accuracy of SNB in patients with breast cancer who were treated with NAC in our series.

\section{Patients and methods}

Seventy patients with breast carcinoma who had been treated with NAC were enrolled in the current study during the period between March 2001 and June 2005. NAC was performed preoperatively and consisted of three to four times of CAF chemotherapy (each chemotherapy administration consisted of $600 \mathrm{mg} / \mathrm{m}^{2}$ of cyclophosphamide, $600 \mathrm{mg} / \mathrm{m}^{2}$ of 5-fluorouracil, and $20-40 \mathrm{mg} /$ body of pirarubicin, every two weeks) (2). 
Table I. Distribution of the pre-NAC stage in breast cancer cases whose sentinel node was identified after NAC according to whether AxLND was performed regardless of an intraoperative sentinel node diagnosis, or not performed because of a negative sentinel node diagnosis.

$\begin{array}{lll}\begin{array}{l}\text { Pre-NAC } \\ \text { stage }\end{array} & \begin{array}{c}\text { AxLND } \\ \text { performed }\end{array} & \begin{array}{l}\text { AxLND not performed because of } \\ \text { a negative sentinel node diagnosis }\end{array}\end{array}$

\begin{tabular}{lrr}
\hline I & 2 & 7 \\
IIA (T2N0) & 14 & 12 \\
IIA (T1N1) & 2 & 0 \\
IIB & 12 & 0 \\
IIIA & 13 & 1 \\
Total & 43 & 20 \\
\hline
\end{tabular}

The number of NAC administrations was decided according to the effect of NAC on the shrinkage of the main breast tumor and metastatic lymph nodes (2). Moreover, the intraarterial (subclavian artery and internal mammary artery) infusion of epirubicin (40 mg) and 5-fluorouracil (1000 mg) was added once to three times preoperatively to patients with large breast tumors or bulky axillary lymph node metastases (N2) for the purpose of performing breast-conserving surgery or controlling metastatic bulky lymph nodes. The number of intra-arterial infusion chemotherapies was also decided according to the effect of chemotherapy. Postoperative systemic chemotherapy was performed the same number of times as systemic NAC or more according to the NAC regimen. Informed consent was obtained from each patient. Intra-arterial infusion chemotherapy was not performed when informed consent could not be obtained. The distribution of the pre-NAC stage of patients whose sentinel node was identified is summarized in Table I. The axillary lymph node status was collectively evaluated by palpation, thin-section computed tomography (CT) and axillary ultrasonography (US) (3). Dynamic MRI findings (4) were also used to evaluate the main breast cancer in addition to the CT and US findings.

SNB was performed using the blue dye method. Under general anesthesia, immediately before surgery a total of $4 \mathrm{ml}$ blue dye (0.4\% Indigocarmine; Daiichi Pharmaceutical, Tokyo, Japan) was injected into four subdermal sites surrounding the tumor (peritumoral injection), and the injection sites were manually massaged for approximately 5-7 min. A sentinel node was defined as a lymph node either that was stained partially or completely by blue dye or which was connected to a blue stained afferent lymphatic tract. After the extirpation of a sentinel node, AxLND was performed in all patients except those who refused AxLND. We always preoperatively explain that AxLND is a standard procedure in breast cancer operation, but 20 out of 37 patients whose axillary lymph node status had been diagnosed to be negative for metastasis before NAC refused AxLND (back-up dissection) when the sentinel node was diagnosed to be metastasis-free intraoperatively. As a result, back-up dissection was performed in 43 patients. A 77-year old female who had been evaluated to be stage III A
Table II. Characteristics of patients who underwent SNB and AxLND after NAC $(n=43)$.

\begin{tabular}{llc}
\hline Age (years) & Mean & 50.0 \\
& Range & $28-69$
\end{tabular}

$\begin{array}{llr}\text { Tumor classification } & & \\ \text { Before NAC } & \text { Tl } & \\ \text { T2 } & \text { T3 } \\ \text { After NAC } & \text { T4 } & \\ & \text { pT0 } & \\ & \text { pTis } & \\ \text { pT1 } & \text { pT2 } \\ & \text { pT3 } & \\ & \text { pT4 }\end{array}$

Lymph node status Before NAC NO 17 N1 21

N2a (fixed axillary nodes) $\quad 5$

After NAC pN0
pN1a (1-3
axillary nodes)
pN2a (4-9
axillary nodes)
pN3a ( $\geq 10$ axillary nodes) 3

Tumor location

Upper outer quadrant $\quad 18$

Lower outer quadrant $\quad 7$

Upper inner quadrant $\quad 12$

Lower inner quadrant 3

Central

4

29

10

0

1

22

15

4
0

0

7

1

5

23

12

5

3

Intra-arterial infusion of epirubicin and 5-FU

None

Once 11

Twice $\quad 8$

Three times $\quad 5$

Surgery of the breast

Breast-conserving surgery $\quad 36$

Mastectomy $\quad 7$

(T3N1) before NAC and whose sentinel node was diagnosed to be metastasis-free intraoperatively and postoperatively after undergoing NAC three times was thus able to avoid AxLND at her request.

For histological examination of the lymph nodes, two or three paraffin sections of a sentinel node and one representative section of non-sentinel nodes were examined using H\&E staining. 
Table III. Comparison of the lymph node status of sentinel nodes and non-sentinel nodes $(n=43)$.

\begin{tabular}{lcc}
\hline & \multicolumn{2}{c}{ Non-sentinel node status } \\
\cline { 2 - 3 } Sentinel node status & Positive & Negative \\
\hline Positive & 11 & 8 \\
Negative & 1 & 23 \\
\hline
\end{tabular}

False-negative rate: $5 \%$.

Table IV. Comparison of the lymph node status of sentinel nodes and non-sentinel nodes in patients with clinically negative axilla before and after NAC $(n=17)$.

\begin{tabular}{lcc}
\hline & \multicolumn{2}{c}{ Non-sentinel node status } \\
\cline { 2 - 3 } Sentinel node status & Positive & Negative \\
\hline Positive & 0 & 4 \\
Negative & 0 & 13 \\
\hline
\end{tabular}

False-negative rate: $0 \%$.

Table V. Comparison of the lymph node status of sentinel nodes and non-sentinel nodes in patients with clinically positive axilla before NAC $(n=26)$.

\begin{tabular}{lcc}
\hline & \multicolumn{2}{c}{ Non-sentinel node status } \\
\cline { 2 - 3 } Sentinel node status & Positive & Negative \\
\hline Positive & 11 & 4 \\
Negative & 1 & 10 \\
\hline
\end{tabular}

False-negative rate: $6.3 \%$.

\section{Results}

The sentinel nodes were successfully identified in 63 out of 70 patients (identification rate: $90 \%$ ). Recently, the sentinel nodes were successfully identified in the most recent 35 patients in a series of 70 patients (identification rate: $100 \%$ for all 35 cases). The mean number of sentinel nodes removed per patient was 1.5 (range 1-6). After SNB an AxLND (backup dissection) was performed in 43 cases, because 20 patients whose axillary lymph node status had been diagnosed to be metastasis negative before NAC refused to undergo AxLND after an intraoperative pathological diagnosis of sentinel node metastasis-free. The characteristics of the 43 patients are summarized in Table II. Of the 43 patients in whom sentinel nodes were identified, 19 (44.2\%) had positive sentinel nodes. For 8 of those 19 patients $(42.1 \%)$, the sentinel node was the only cancer positive lymph node. Among the 24 patients who had negative sentinel nodes it was found that one patient had a confirmed false negative result, thus yielding a false negative rate of $5 \%$, sensitivity of $95 \%$ (Table III).

In the subgroup of patients with a clinically negative lymph node status (N0) before NAC (17 patients), no false negative patients were observed (Table IV), whereas in the subgroup of patients with a clinically positive lymph node status (N1, N2) before NAC (26 patients), the false negative rate was $6.3 \%$ (Table $\mathrm{V}$ ). It was thus suggested that clinically positive lymph node patients before NAC tended to yield false negative results.

\section{Discussion}

Because neoadjuvant chemotherapy (NAC) not only increased the rate of breast-conserving surgery in locally advanced breast cancer patients, but also completely cleared invasive cancer cells in the breast tumor and metastatic axillary lymph nodes in some cases, NAC has now become a standard treatment of locally advanced breast cancer, and it has quickly obtained an important position in breast cancer treatment (5). NAC is now recognized to be valuable for determining the treatment strategy for each breast cancer patient, not only in advanced cases but also in early cases, because NAC discloses an in vivo evaluation of breast cancer sensitivity to particular anti-cancer agents. As a result, it has been discussed that SNB could enable us to avoid AxLND in cases that respond to NAC. However, there is still limited data on SNB after NAC.

Controversy remains regarding the feasibility and accuracy of SNB after NAC, because NAC may damage the lymphatic drainage, particularly in locally advanced breast cancer cases, thus causing lymphatic scarring after tumor cell necrosis of the metastatic lymph nodes and lymphatic routes, and also resulting in changes in lymphatic drainage.

Two studies have reported a high false-negative rate $(6,7)$ associated with SNB for patients with breast cancer who had been treated with NAC, while other studies (8-11) report an acceptable false-negative rate. In the NSABP B-27 protocol trial, SNB was performed in 428 patients treated with NAC, $76 \%$ of which were axillary lymph node status N0 and $63 \%$ had a breast tumor size of smaller than $4 \mathrm{~cm}$, and the trial reported an $85 \% \mathrm{SN}$ identification rate and a $10 \%$ falsenegative rate (11). These widely varied false-negative rates are supposed to be the consequence of the difference in the enrolled cases between these studies. Shimazu et al (12) performed SNB by combination method with radiocolloid and blue dye in 47 patients who were treated with NAC, and reported an acceptable SN identification rate (94\%), but a high false-negative rate $(15 \%)$ in the subgroup of patients with a clinically positive lymph node status before and/or after NAC, whereas they reported an acceptable false-negative rate $(7 \%)$ in the subgroup of patients with a clinically negative lymph node status both before and after NAC, thus suggesting that SNB cannot predict the axillary lymph node status of the patients with a clinically positive lymph node status before and/or after NAC. 
Our study included not only patients who were treated with systemic CAF chemotherapy but also patients with a lymph node status of N2 before NAC who were treated with systemic CAF and intra-arterial infusion chemotherapy (epirubicin and 5-FU) which could potentially cause inflammation of the local area. In the procedure of AxLND of cases which were treated with intra-arterial infusion targeting the metastatic axillary lymph nodes, adhesion was observed between the vessels and fatty tissue containing lymph nodes, and the skeltonization of the vessels was more difficult than in cases which were not treated with intra-arterial infusion. Nevertheless, the $90 \%$ SN identification rate and $5.0 \%$ false-negative rate in our series were comparable to the rate reported for early-stage breast cancer patients who were not treated with NAC. We experienced two cases whose axillary bulky lymph node metastasis (N2) before NAC disappeared after NAC, and metastasis was proven in the sentinel node only by postoperative pathological diagnosis. Kuerer et al (13) speculate that SNB shows a reliable accuracy in patients who have multiple metastatic lymph nodes only when the sentinel node and non-sentinel nodes respond identically to NAC.

In our series, both serial H\&E sectioning and immunohistochemical analysis were not used to diagnose metastasis of the sentinel nodes pathologically. Kuerer et al (13) reported that the occult metastasis detected by serial H\&E sectioning or anti-cytokeratin immunohistochemical staining was proven in four lymph nodes from four patients who corresponded to $10 \%(4 / 39)$ of the cases which had been diagnosed to be metastasis-negative by a single H\&E stained section of the dissected axillary lymph nodes (median number: 16/patient) after NAC, but had been diagnosed metastasis-positive by cytology before NAC. The detection rate of micrometastasis by either serial H\&E sectioning or immunohistochemical staining has been reported (14-16) to range from $9 \%$ to $13 \%$ in patients not initially treated with NAC. If further studies prove a higher detection rate of micrometastasis in the sentinel node diagnosed to be metastasis-free in a group of patients treated with NAC than that of micrometastasis in a group of patients not-treated with NAC, SNB would not play a significant role in patients treated with NAC. However, if further studies can prove the same detection rate of micrometastasis in NAC patients as that of patients nottreated with NAC, then SNB after NAC can play the same diagnostic role as SNB in patients not treated with NAC.

The biggest advantage of NAC is that it can help to clarify the cancer characteristics of sensitivity to anti-cancer agents in vivo and this is a superior point in comparison to postoperative chemotherapy. In many institutes, NAC is limited to locally advanced breast cancer patients treated with larger doses of anthracycline-agents than our regimen, but we consider that a clinical and pathological response can be achieved not only in advanced cases but also in early breast cancer cases in spite of our lower dose of anthracycline-agent than that used in other studies. In this context, performing NAC in non-advanced breast cancer patients is recognized to be valuable for determining the treatment strategy for each breast cancer patient, and also results in an increased rate of breast-conserving surgery. Patients who have had their axillary lymph node status downstaged to N0 from metastasis-positive $\mathrm{N} 1$ and $\mathrm{N} 2$ are not appropriate candidates for avoiding AxLND after negative SNB because there is no assurance that the sentinel nodes and non-sentinel nodes respond identically to NAC. However, the diagnosis of axillary lymph node status has recently become more accurate thanks to the use of thin-section computed tomography (CT) and ultrasonography (US). In patients who were diagnosed to be axillary lymph node metastasis-negative by CT and US before NAC, but were not recognized as candidates for SNB according to conventional criteria due to large-sized breast tumors, for example T2N0 cases, because of the higher possibility of lymph node metastasis than T1N0 cases, and are diagnosed by intraoperative pathology to be sentinel node metastasis-negative after NAC, we hypothesize that the sentinel node was metastasis-free before NAC or the micrometastasis of the sentinel node was removed by NAC. According to Shimazu et al (12) and our study, the false negative rate in the subgroup of patients with a clinically negative lymph node status before NAC is supposed to be the same level as the false negative rate of early breast cancer cases.

If pCR is achieved in the metastatic axillary lymph nodes, AxLND can thus theoretically be ruled out by SNB. Kuerer et al (13) reported that $72 \%(23 / 32)$ of the cases whose main breast cancer converted to pCR after NAC achieved a conversion to $\mathrm{pCR}$ in the metastatic lymph nodes. In the NSABP B-18 trial (5), 185 patients whose axillary lymph nodes were metastasis positive, were treated with NAC, and in $40 \%$ of these patients, pCR of axillary lymph node metastasis was achieved, whereas pCR of both main breast cancer and axillary lymph node metastasis was achieved in only $7 \%$ of the patients. As a result, it is supposed that pCR is achieved in the metastatic axillary lymph nodes previous to in main breast cancer in the majority of cases. The cases whose main breast cancer converted to $\mathrm{pCR}$ should thus be considered to be candidates for SNB because the possibility of pCR in the axillary lymph nodes is high.

In conclusion, SNB after NAC is thus considered to be a feasible and applicable modality for patients with clinically negative lymph node status (N0) before NAC. Further studies are necessary regarding the performance of SNB in patients who have had their axillary lymph node status downstaged from clinically positive $(\mathrm{N} 1, \mathrm{~N} 2)$ to clinically negative (N0) and patients who achieved pCR in their main breast tumors.

\section{References}

1. Kuerer HM, Newman LA, Smith TL, et al: Clinical course of breast cancer patients with complete pathologic primary tumor and axillary lymph node response to doxorubicin-based neoadjuvant chemotherapy. J Clin Oncol 17: 460-469, 1999.

2. Ogawa Y, Nishioka A, Kubota K, et al: CT findings of breast cancer with clinically complete response following neoadjuvant chemotherapy-histological correlation. Oncol Rep 10: 1411-1415, 2003.

3. Ogawa Y, Nishioka A, Nishigawa T, et al: Thin-section CT evaluation and pathologic correlation of therapeutic effect of neoadjuvant chemotherapy for axillary lymph nodes of clinically node-positive breast cancer patients. Oncol Rep 10: 985-989, 2003.

4. Tsuboi N, Ogawa $\mathrm{Y}$, Inomata $\mathrm{T}$, et al: Changes in the findings of dynamic MRI by preoperative CAF chemotherapy for patients with breast cancer of stage II and III: pathologic correlation. Oncol Rep 6: 727-732, 1999.

5. Fisher B, Brown A, Mamounas E, et al: Effect of preoperative chemotherapy on local-regional disease in women with operable breast cancer: findings from National Surgical Adjuvant Breast and Bowel Project B-18. J Clin Oncol 15: 2483-2493, 1997. 
6. Nason KS, Anderson BO, Byrd DR, et al: Increased false negative sentinel node biopsy rates after preoperative chemotherapy for invasive breast carcinoma. Cancer 89: 2187-2194, 2000.

7. Fernandez A, Cortes M, Benito E, et al: Gamma probe sentinel node localization and biopsy in breast cancer patients treated with a neoadjuvant chemotherapy scheme. Nucl Med Commun 22: 361-366, 2001.

8. Breslin TM, Cohen LF, Sahin A, et al: Sentinel lymph node biopsy is accurate after neoadjuvant chemotherapy for breast cancer. J Clin Oncol 18: 3480-3486, 2000.

9. Julian TB, Dusi D and Wolmark N: Sentinel node biopsy after neoadjuvant chemotherapy for breast cancer. Am J Surg 184: 315-317, 2002.

10. Haid A, Tausch C, Lang A, et al: Is sentinel lymph node biopsy reliable and indicated after preoperative chemotherapy in patients with breast carcinoma? Cancer 92: 1080-1084, 2001.

11. Mamounas EP, Brown A, Anderson S, et al: Sentinel node biopsy after neoadjuvant chemotherapy in breast cancer: Results from National Surgical Adjuvant Breast and Bowel Project Protocol B-27. J Clin Oncol 23: 2694-2702, 2005.
12. Shimazu K, Tamaki Y, Taguchi T, Akazawa K, Inoue $\mathrm{T}$ and Noguchi S: Sentinel lymph node biopsy using periareolar injection of radiocolloid for patients with neoadjuvant chemotherapy-treated breast carcinoma. Cancer 100: 2555-2561, 2004.

13. Kuerer HM, Sahin AA, Hunt KK, et al: Incidence and impact of documented eradication of breast cancer axillary lymph node metastasis before surgery in patients treated with neoadjuvant chemotherapy. Ann Surg 230: 72-78, 1999.

14. International (Ludwig) Breast Cancer Study Group: Prognostic importance of occult axillary lymph node micrometastasis from breast cancers. Lancet 335: 1565-1568, 1990.

15. Hainsworth PJ, Tjandra JJ, Stillwell RG, et al: Detection and significance of occult metastasis in node-negative breast cancer. Br J Surg 80: 459-463, 1993.

16. Clare SE, Sener SF, Wilkens W, Goldschmidt R, Merkel D and Winchester DJ: Prognostic significance of occult lymph node metastasis in node-negative breast cancer. Ann Surg Oncol 4: 447-451, 1997. 